\title{
Quantum and Classical Vibrational Relaxation Dynamics of $N$-Methylacetamide on Ab Initio Potential Energy Surfaces
}

\author{
HIROSHI FUJISAKI, ${ }^{1 *}$ KIYOSHI YAGI, ${ }^{2}$ JOHN E. STRAUB, ${ }^{3}$ \\ GERHARD STOCK ${ }^{1}$ \\ ${ }^{1}$ Institute of Physical and Theoretical Chemistry, J. W. Goethe University, Max-von-Laue-Str. 7, \\ 60438 Frankfurt, Germany \\ ${ }^{2}$ Department of Applied Chemistry, School of Engineering, The University of Tokyo, Hongo 7-3-1, \\ Bunkyo-ku, Tokyo 113-8656, Japan \\ ${ }^{3}$ Department of Chemistry, Boston University, 590 Commonwealth Avenue, Boston, MA 02215
}

Received 23 September 2008; accepted 15 December 2008

Published online 23 March 2009 in Wiley InterScience (www.interscience.wiley.com).

DOI 10.1002/qua.22061

\begin{abstract}
Employing extensive quantum-chemical calculations at the DFT/B3LYP and MP2 level, quartic force fields of isolated $N$-methylacetamide are constructed. Taking into account 24 vibrational degrees of freedom, the model is employed to perform numerically exact vibrational configuration interaction calculations of the vibrational energy relaxation of the amide I mode. It is found that the energy transfer pathways may sensitively depend on details of the theoretical description. Moreover, the exact reference calculations were used to study the applicability and accuracy of (i) the quasiclassical trajectory method, (ii) time-dependent second-order perturbation theory, and (iii) the instantaneous normal mode description of frequency fluctuations. Based on the results, several strategies to describe vibrational energy relaxation in biomolecular systems are discussed. (C) 2009 Wiley Periodicals, Inc. Int J Quantum Chem 109: 2047-2057, 2009
\end{abstract}

Key words: quantum dynamics; biomolecule; vibration; relaxation

Correspondence to: H. Fujisaki; e-mail: fujisaki@riken.jp

*Present address: Integrated Simulation of Living Matter Group, Computational Science Research Program, RIKEN, 2-1 Hirosawa, Wako, Saitama 351-0198, Japan.

Dedicated to Prof. Kimihiko Hirao on the occasion of his retirement from the University of Tokyo.

Contract grant sponsor: Alexander von Humboldt Foundation, Frankfurt Center for Scientific Computing, Fonds der Chemischen Industrie. 


\section{Introduction}

A mide modes in peptides and proteins are important probes to establish a structurespectroscopy relation for biomolecules [1, 2]. The amide I modes, which are localized at the $\mathrm{C}=\mathrm{O}$ bond with frequencies of $1,600-1,700 \mathrm{~cm}^{-1}$, have attracted special attention because they are sensitive to the secondary structure of peptides [3]. This work is concerned with the vibrational energy relaxation of amide I modes. Recent experimental studies on model peptides and various small globular peptides have shown that the $v=1 \rightarrow 0$ population decay time $T_{1}$ of the amide I mode is about 1 ps for all systems considered [4-6]. To explain the ultrafast energy and phase relaxation of amide I modes, a number of theoretical formulations have been given [7-20], many of which focus on $\mathrm{N}$-methylacetamide (NMA, $\mathrm{H}_{3} \mathrm{C}-\mathrm{COND}-\mathrm{CH}_{3}$ ), a peptide-like small molecule containing only a single amide I mode. Employing standard biomolecular potential energy functions (such as CHARMM [21] or GROMOS [22]), quasiclassical trajectory simulations [19] and time-dependent perturbation theory calculations [20] have been performed, qualitatively reproducing the subpicosecond relaxation time found for NMA in $\mathrm{D}_{2} \mathrm{O}$ [4-6].

Alternatively, various groups considered $a b$ initio based potential energy functions to describe the dynamics of amide I vibrations [7-14, 16, 17]. Employing density functional theory (DFT) calculation at the B3LYP/6-31G+(d) level, we recently constructed a quartic force field of isolated NMA [16]. The dynamics of the amide I vibration was studied using the vibrational configuration interaction method [16, 23-25], including 24 vibrational degrees of freedom. The study indicated that the energy transfer pathways may sensitively depend on details of the theoretical description. Hence, one goal of the present paper is to examine the sensitivity of amide I relaxation with respect to the ab initio level (DFT/B3LYP and MP2), basis set $(6-31 \mathrm{G}+(\mathrm{d})$ and $6-31 \mathrm{G}++(\mathrm{d}, \mathrm{p}))$, and description of the solvent (none or continuum model). Note that "relaxation" means intramolecular one in this paper, and we do not explicitly consider the effect of baths. In the full quantum calculations below, an initial state is one of basis configurations (a single VSCF configuration, see below), which is assumed to be a bright state created by a laser pulse, and the relaxation is represented by "mixing" among such basis configurations.
As the vibrational configuration interaction method gives a numerically exact quantummechanical description of the vibrational dynamics of the model system, it provides an ideal means to test several approximations usually employed in the description of vibrational energy relaxation (Numerically exact quantum calculations of multidimensional vibrational energy redistribution have been also performed using the MCTDH method; see for example [26]). The focus of this work is therefore to study the applicability and accuracy of (i) the quasiclassical trajectory method [19], (ii) (time-dependent) second-order perturbation theory $[20,27]$, and (iii) the instantaneous normal mode description [28] of frequency fluctuations. Based on the results, we discuss several strategies to describe vibrational energy relaxation in biomolecular systems.

\section{Theory and Methods}

\subsection{QUARTIC FORCE FIELD}

Although there are various ways to construct ab initio potential energy surfaces (PES) $[29,30]$, we employ here a simple but accurate method called quartic force field, which is useful if we consider vibrational dynamics around a single equilibrium state of a molecule [31-33]. In this approach, the PES is approximated by a fourth-order Taylor expansion around the equilibrium geometry

$$
\begin{aligned}
\tilde{V}\left(\left\{Q_{i}\right\}\right)=\frac{1}{2} \sum_{k} \omega_{k}^{2} Q_{k}^{2} & +\frac{1}{3 !} \sum_{k, l, m} t_{k l m} Q_{k} Q_{l} Q_{m} \\
& +\frac{1}{4 !} \sum_{k, l, m, n} u_{k l m n} Q_{k} Q_{l} Q_{m} Q_{n},
\end{aligned}
$$

where $Q_{k}$ and $\omega_{k}$ denote the $k$ th normal coordinate and the associated harmonic frequency, respectively, and the coefficients $t_{k l m}$ and $u_{k l m n}$ represent the third- and fourth-order derivatives of the PES. The aforementioned expression can be recast in the form of the $n$-mode coupling representation $(n \mathrm{MR})$ [33-35]

$$
\begin{gathered}
V\left(\left\{Q_{i}\right\}\right)=V^{1 \mathrm{MR}}\left(\left\{Q_{i}\right\}\right)+V^{2 \mathrm{MR}}\left(\left\{Q_{i}\right\}\right) \\
+V^{3 \mathrm{MR}}\left(\left\{Q_{i}\right\}\right), \quad(2)
\end{gathered}
$$




$$
\begin{aligned}
V^{2 \mathrm{MR}}\left(\left\{Q_{i}\right\}\right)=\sum_{i \neq j}\left(\frac{1}{2} t_{i j j} Q_{i} Q_{j}^{2}\right. & \left.+\frac{1}{6} u_{i j j j} Q_{i} Q_{j}^{3}\right) \\
& +\sum_{i<j} \frac{1}{4} u_{i i j j} Q_{i}^{2} Q_{j}^{2}, \quad(4) \\
V^{3 \mathrm{MR}}\left(\left\{Q_{i}\right\}\right)=\sum_{i<j<k} t_{i j k} Q_{i} Q_{j} Q_{k} & +\sum_{i \neq j<k} \frac{1}{2} u_{i j k} Q_{i}^{2} Q_{j} Q_{k} .
\end{aligned}
$$

Note that we have omitted the $4 \mathrm{MR}$ terms $V^{4 \mathrm{MR}}\left(\left\{Q_{i}\right\}\right)=\sum_{i<j<k<l} u_{i j k l} Q_{i} Q_{j} Q_{k} Q_{l}$. This strategy has been successfully applied to several molecules [35-38]. By adding the normal mode kinetic energy $K=\sum_{k} P_{k}^{2} / 2$ (we neglect the so-called Coriolis and Watson terms [25] because we restrict ourselves to small-amplitude motions in a large molecule), we obtain the vibrational Hamiltonian $H=K+V\left(\left\{Q_{i}\right\}\right)$ for the system.

In this study, the quartic force field was derived using the B3LYP or MP2 methods with the 6$31 \mathrm{G}+(\mathrm{d})$ or $6-31 \mathrm{G}++(\mathrm{d}, \mathrm{p})$ basis set. First, the equilibrium structure and the harmonic frequencies were obtained for trans-NMA using the Gaussian03 program package [39]. Then, the third and fourth-order derivatives were calculated by numerical differentiation of the analytic Hessian [33] using the Sindo code developed by Yagi [40] or by the "anharmonic" option of Gaussian 03.

\subsection{VIBRATIONAL CONFIGURATION INTERACTION METHOD}

In a first step, we calculated the vibrational energies and eigenstates of the above described model of NMA using the vibrational configuration interaction (VCI) method developed by Bowman and coworkers [25]. To generate basis functions for the VCI calculations, we first performed a vibrational selfconsistent field (VSCF) calculation of the vibrational ground state. The VSCF wave function is expressed as a direct product of one-mode functions or modals [41] as

$$
\Phi_{\mathbf{n}}^{\mathrm{VSCF}}=\prod_{i=1}^{f} \phi_{n_{i}}^{(i)}\left(Q_{i}\right),
$$

where $\mathbf{n}$ and $f$ denote the vibrational quantum numbers and the number of degrees of freedom, respectively. The modals are determined by the VSCF equation

$$
\left[-\frac{1}{2} \frac{\partial^{2}}{\partial Q_{i}^{2}}+\left\langle\prod_{j \neq i} \phi_{n_{j}}^{(j)}|V| \prod_{j \neq i} \phi_{n_{j}}^{(j)}\right\rangle\right] \phi_{n_{i}}^{(i)}=\epsilon_{n_{i}}^{(i)} \phi_{n_{i}}^{(i)},
$$

which yields the vibrational ground state $(\mathbf{n}=\mathbf{0})$ and the virtual modals. The VCI wave function is expressed as a linear combination of VSCF configurations

$$
\Psi_{\mathbf{n}}^{\mathrm{VCI}}=\sum_{\mathbf{m}} C_{\mathrm{mn}} \Phi_{\mathrm{m}}^{\mathrm{VSCF}}
$$

The VCI wave functions and energy levels are obtained by diagonalization of the VCI matrix

$$
H_{\mathrm{mn}}=\left\langle\Phi_{\mathbf{m}}^{\mathrm{VSCF}}|H| \Phi_{\mathbf{n}}^{\mathrm{VSCF}}\right\rangle .
$$

All VSCF/VCI calculations were carried out using the Sindo code [40] for nonrotating molecules. The modals were expanded in terms of harmonic oscillator wave functions. The number of wave functions employed were $11,9,7$, and 5 for $\left\{\phi^{(7)}, \phi^{(8)}\right\},\left\{\phi^{(9)}\right.$ $\left.\phi^{(12)}\right\},\left\{\phi^{(13)}-\phi^{(23)}\right\}$, and $\left\{\phi^{(24)}-\phi^{(30)}\right\}$, respectively. The mode index was labeled in increasing order of the frequency and the six lowest-lying modes were kept frozen. The VSCF configurations were selected by increasing energy until a cut-off energy of $\sim 5,000$ $\mathrm{cm}^{-1}$, resulting in $N_{\mathrm{CI}} \simeq 6,000$.

Once all eigenvalues $\left\{E_{\mathbf{n}}\right\}$ and eigenfunctions $\left\{\Psi_{\mathrm{n}}^{\mathrm{VCI}}\right\}$ are obtained, it is straightforward to calculate the time-dependent wave function

$$
|\Psi(t)\rangle=\sum_{\mathbf{n}}\left\langle\Psi_{\mathbf{n}}^{\mathrm{VCI}} \mid \Psi(0)\right\rangle e^{-i E_{\mathbf{n}} t / \hbar}\left|\Psi_{\mathbf{n}}^{\mathrm{VCI}}\right\rangle,
$$

which represents a numerically exact description of the aforementioned quartic force field model.

In all calculations discussed below we assume that the initial state is given by a single VSCF configuration $\Psi(0)=\Phi_{i}^{\mathrm{VSCF}}$, representing the $n=1$ state of the amide I vibration. We thus obtain for the wave function

$$
|\Psi(t)\rangle=\sum_{\mathbf{j}} O_{\mathbf{j}}(t)\left|\Phi_{\mathbf{j}}^{\mathrm{VSCF}}\right\rangle
$$

with

$$
O_{\mathbf{j}}(t)=\sum_{\mathbf{n}} C_{\mathbf{j} \mathbf{n}} C_{\mathbf{n} \mathbf{i}} e^{-i E_{\mathbf{n}} t / \hbar}
$$

and $P_{\mathbf{j}}(t)=\left|O_{\mathbf{j}}(t)\right|^{2}$ for the time-dependent probability of state $\Phi_{j}^{\mathrm{VSCF}}$. This allows us to define the harmonic energy of vibrational mode $i$ as

$$
E_{i}(t)=\hbar \omega_{i} \sum_{\mathbf{j}} P_{\mathbf{j}}(t) n_{\mathbf{j}}^{(i)}
$$


where the zero point energy of the mode was disregarded. Equation (13) will be used below to discuss the vibrational energy relaxation in NMA.

\subsection{TIME-DEPENDENT PERTURBATION THEORY}

Employing time-dependent perturbation theory, recently Fujisaki et al. have extended Fermi's Golden Rule to the non-Markovian regime [20, 42]. As a stringent test of this formulation, we wish to compare the perturbative results to the exact VCI results obtained for NMA. Assuming zero temperature, the perturbative expression for the amide I ground state population is given by [20]

$$
\begin{aligned}
P_{0}(t) & =\rho_{00}(t) \simeq \frac{\hbar}{8 \omega_{S}} \sum_{\alpha, \beta} \frac{t_{S \alpha \beta}^{2}}{\omega_{\alpha} \omega_{\beta}} \frac{1-\cos \left(\omega_{S}-\omega_{\alpha}-\omega_{\beta}\right) t}{\left(\omega_{S}-\omega_{\alpha}-\omega_{\beta}\right)^{2}} \\
& \equiv \sum_{\alpha, \beta} F_{S \alpha \beta}^{2}\left[1-\cos \left(\omega_{S}-\omega_{\alpha}-\omega_{\beta}\right) t\right]
\end{aligned}
$$

where $\omega_{S}$ is the system frequency, $\omega_{\alpha}$ denote the (harmonic) frequency of the bath modes, and $t_{S \alpha \beta}$ represents the third-order coupling elements [20]. Moreover, we have introduced a Fermi resonance parameter [43]

$$
\begin{aligned}
F_{S \alpha \beta} & \equiv\left|\sqrt{\frac{\hbar}{2 \omega_{S}} \frac{\hbar}{2 \omega_{\alpha}} \frac{\hbar}{2 \omega_{\beta}}} \frac{t_{S \alpha \beta}}{\hbar\left(\omega_{S}-\omega_{\alpha}-\omega_{\beta}\right)}\right| \\
& \equiv\left|\frac{\tau_{S \alpha \beta}}{\omega_{S}-\omega_{\alpha}-\omega_{\beta}}\right|
\end{aligned}
$$

which is proportional to

$$
\eta \equiv\left|\frac{\langle i|\Delta V| f\rangle}{\Delta E}\right|,
$$

where $|i\rangle$ and $|f\rangle$ are the initial and final harmonic states, $\Delta V=V-\sum_{k} \omega_{k}^{2} Q_{k}^{2} / 2$ denotes the anharmonic vibrational coupling, and $\Delta E$ is the energy difference between $|i\rangle$ and $|f\rangle$. The Fermi resonance parameter is a key ingredient in the interpretation of vibrational energy transfer processes [20]. This concept has been useful for the classical dynamics characterization of the vibrational relaxation pathways in water [44] and in myoglobin [45], although there may be differences in the mechanism of quantum and classical energy transfer [46].

\subsection{CLASSICAL DESCRIPTION}

Quasiclassical trajectory calculations are a wellestablished approach to approximately calculate the energy redistribution in molecular systems [47]. In this method, the initial state of the quantum system (e.g., the $n=1$ state prepared by an infrared laser pulse) is represented by a phase-space distribution (e.g., the Wigner distribution), which is sampled by an ensemble of classical trajectories. Starting by construction with the correct initial state and recalling that classical mechanics can be considered as a short-time approximation to quantum mechanics, a classical simulation should be a good approximation to quantum-mechanics in the case of ultrafast relaxation dynamics [48].

In this work, we solved Newton's equation on the PES defined in Eq. (1), using Yoshida's sixth-order symplectic integrator with a 0.5 fs time step [49]. To generate classical initial conditions for the positions and momenta, we represent the normal modes $\left\{Q_{k}, P_{k}\right\}$ in terms of classical action-angle variables $\left\{n_{k}, \phi_{k}\right\}[47]$

$$
\begin{aligned}
Q_{k} & =\sqrt{\left(2 n_{k}+\gamma\right) \hbar / \omega_{k}} \sin \phi_{k}, \\
P_{k} & =\sqrt{\left(2 n_{k}+\gamma\right) \hbar \omega_{k}} \cos \phi_{k},
\end{aligned}
$$

where the factor $\gamma=1$ accounts for the zero-point energy of the mode. To obtain the initial positions and momenta of the initially excited amide I mode, we associate the action $n_{k}$ with the initial quantum state of the amide I mode, i.e., $n_{k}=1$ for the first excited state. The remaining modes are for $T=0$ $\mathrm{K}$ initially in the ground state $n_{k}=0$ and only vibrate according to their zero-point energy. In all cases, the vibrational phases $\phi_{k}$ are picked randomly from the interval $[0,2 \pi]$. Using this initial conditions, the classical energy content of vibrational mode $i$ is defined as

$$
E_{i}(t)=\frac{\left\langle P_{i}^{2}(t)\right\rangle}{2}+\frac{\omega_{i}^{2}}{2}\left\langle Q_{i}^{2}(t)\right\rangle-E_{i}^{\mathrm{ZPE}},
$$

where $\langle\ldots\rangle$ represents an ensemble average over 100 nonequilibrium trajectories and $E_{i}^{\mathrm{ZPE}}$ denotes the zero-point energy of mode $i$.

Although Eqs. (17) and (18) represent a correct quasiclassical representation of the initial state of the system (in the sense that the vibrational energy distribution is correct), it may give rise to unphysical behavior due to the well-known zero-point energy problem of classical mechanics [50]. In quantum mechanics, each oscillator mode must hold an 
amount of energy that is larger or equal to the zero-point energy of this mode. In a classical trajectory calculation, on the other hand, energy can flow among the modes without this restriction. In the case of our ab initio model of NMA, for example, we have found that the high zero-point energy contained in the $\mathrm{C}-\mathrm{H}$ stretch modes $\left(\approx 1600 \mathrm{~cm}^{-1}\right)$ may be transferred to a large amount to low-frequency modes of NMA, thus yielding unphysical and unstable trajectories.

Various approaches have been proposed to fix the zero-point energy problem [50], however, most of these techniques share the problem that they manipulate individual trajectories, whereas the conservation of zero-point energy should correspond to a virtue of the ensemble average of trajectories. Here, we adopt the method introduced in Ref. 51, which invokes quantum corrections to the classical calculation in order to restrict the classically accessible phase space according to the rules of quantum mechanics. At the simplest level of the theory, these corrections have been shown to correspond to including only a fraction $\gamma(0 \leq \gamma \leq 1)$ of the full zero-point energy into the classical calculation. The quantum correction $\gamma$ can be determined by requiring that some observables of the system are reproduced [51], e.g., that the amide I energy remains larger than the zero-point energy for all times under consideration. In the present case, we assigned full zero-point energy to the initially excited amide I mode, while for the remaining modes we choose $\gamma$ according to the classical thermal energy at $T=300$ $\mathrm{K}$. This protocol is simple to implement in standard MD codes and was shown to give good results in previous quasiclassical trajectory studies of vibrational energy redistribution [19].

\section{Results and Discussion}

\subsection{QUANTUM DYNAMICS AT VARIOUS LEVELS OF AB INITIO THEORY}

The theoretical description of the amide I vibration of NMA first of all depends on the quantumchemical parameterization of the quartic force field. Here, the following four levels of theory were employed: DFT/B3LYP with 6-31+G(d) or 6$31++G(d, p)$ basis set and MP2 with $6-31+G(d)$ or $6-31++G(d, p)$ basis set. To facilitate the comparison to experiment, we first consider the vibrational frequencies of NMA, as obtained from the diagonalization of the VCI matrix (9). Figure 1 compares these frequencies to experimental values obtained under argon matrix conditions [52], focusing on vibrational modes between 1,000 and $2,000 \mathrm{~cm}^{-1}$. MP2 somewhat overestimates the anharmonic frequencies in comparison to DFT/B3LYP. Applying the $6-31+\mathrm{G}(\mathrm{d})$ basis set, we obtain an overall deviation (mean absolute deviation) of 52 and $26 \mathrm{~cm}^{-1}$ at the MP2 and DFT/B3LYP level, respectively. As the improvement due to the larger $6-31++G(d, p)$ basis is relatively small (compared to the increased effort), in the following we only show results using the 6$31+G(d)$ basis. We confirmed that the energy transfer pathways are nearly the same in both cases.

Although all levels of theory at least qualitatively reproduce the vibrational frequencies of NMA, the deviations may be more significant when the vibrational energy relaxation of the amide I vibration is considered. Figure 2 shows the time evolution of the energy content [Eq. (13)] of the initially excited amide I mode (\# 23) as well as of the remaining modes of the molecule. As discussed previously [16], in the B3LYP / 6-31G+ $(\mathrm{d})$ calculation most of the amide energy goes to the 9 th vibrational mode at $869 \mathrm{~cm}^{-1}$ via the pathway $\left|23_{1}\right\rangle \rightarrow\left|9_{2}\right\rangle$ and to the 7 th and 12 th modes via the pathway $\left|23_{1}\right\rangle \rightarrow\left|7_{1} 12_{1}\right\rangle$. As discussed in Ref. 16, these normal modes correspond to motion of the C-terminal methyl-group atoms. The pathways can be explained by considering the Fermi resonance parameter $F_{S \alpha \beta}$ defined in Eq. (15), which describes within time-dependent perturbation theory the intensity of transition $\left|S_{1}\right\rangle \rightarrow\left|\alpha_{1} \beta_{1}\right\rangle$. Figure 3 shows the Fermi resonance parameters for all mode combinations $(\alpha, \beta)$ together with its resonance factor $\left|1 /\left(\omega_{S}-\omega_{\alpha}-\omega_{\beta}\right)\right|$ and the anharmonic coefficient $\left|\tau_{S \alpha \beta}\right|$. Interestingly, it is the resonance factor rather than the anharmonic coefficient that determines $F_{S \alpha \beta}$ and thus the relaxation pathway, i.e., the shape of $\left|F_{S \alpha \beta}\right|$ mainly reflects that of $\left|1 /\left(\omega_{S}-\omega_{\alpha}-\omega_{\beta}\right)\right|$. This underlines the importance of an accurate calculation of vibrational frequencies. Another point is that $\left|\tau_{S \alpha \beta}\right|$ is nearly equal for both calculation levels (DFT or MP2). This fact is the basis for multiresolution methods to calculate vibrational frequencies by combining high and low level quantum-chemical methods [30, 31].

Employing the force field obtained from the MP2/6-31G+(d) calculations, Figure 2 shows a qualitative change of the amide I relaxation dynamics as compared to the results at the DFT/B3LYP level. Although the same vibrations are involved in the process (i.e., mainly modes \# 9, and \# 7 and \# 12), the amide I energy decreases only by $\approx 20 \%$ instead of $\approx 80 \%$. Figure 3 reveals that this is caused by the fact 


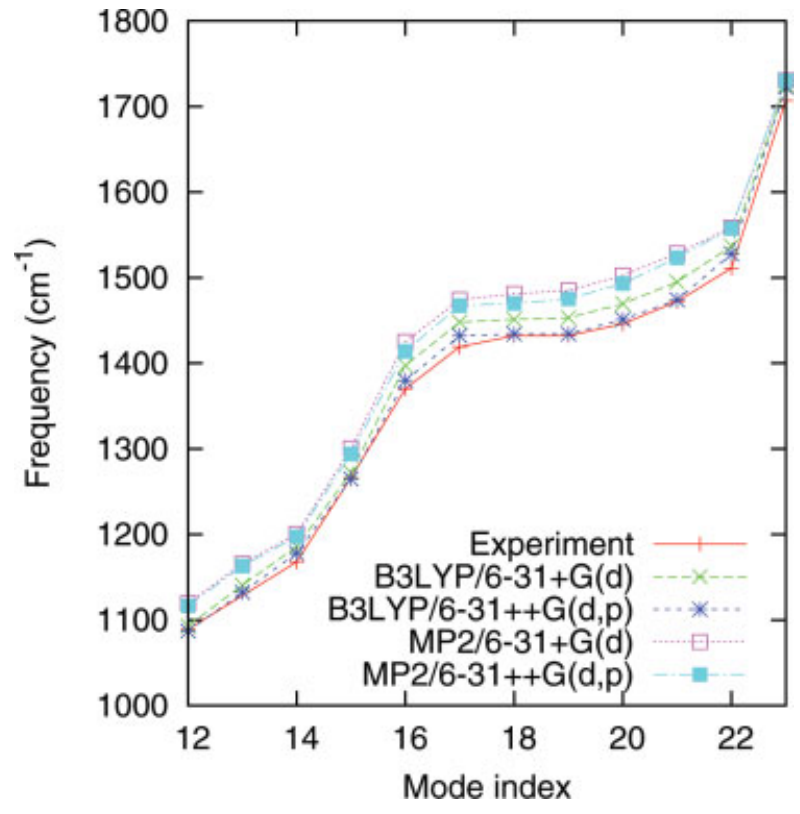

FIGURE 1. Vibrational frequencies of isolated $\mathrm{N}$-methylacetamide (NMA), calculated from the quartic force field using the VSCF/VCI method. Compared are experimental [52] and calculated frequencies at various levels of theory. The amide I vibration corresponds to mode \# 23 and amide II to \# 22. [Color figure can be viewed in the online issue, which is available at www.interscience.wiley.com.]

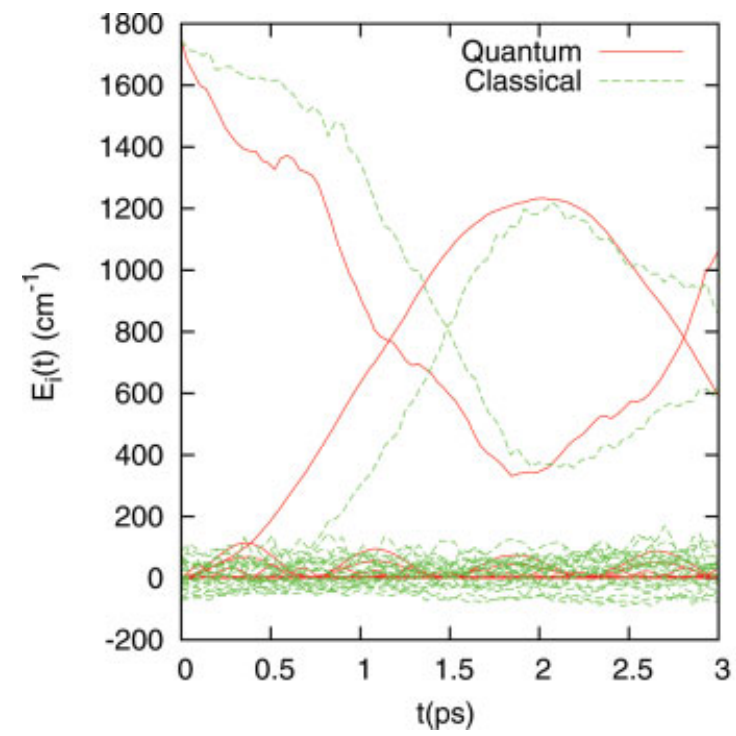

that the value of the corresponding Fermi resonance parameters for MP2 are only half of the value of the DFT parameters. (Note that $F_{S \alpha \beta}$ enters quadratically in the relaxation rate, which explains the overall factor of four.)

As an approximative way to include solvent effects into the parameterization of the quartic force field, we have employed the implicit solvent model as implemented in Gaussian03 [39] and repeated the aforementioned calculations. As an example, Figure 4 shows results obtained for implicit water and implicit methanol at the DFT/B3LYP level of theory. In both cases, one obtains a relatively weak amide I relaxation, which mainly involves the modes $\# 7$, \#8, \#11, and \#12. However, it should be stressed that in experiment $[4,5]$ as well as in simulations using explicit water solvent $[19,20]$ there is a strong flow of vibrational energy into the solvent degrees of freedom which is, of course, not captured by an implicit model. Hence, one should regard these calculations as another example of energy transfer dynamics on different PESs.

\subsection{CLASSICAL RELAXATION DYNAMICS}

The numerically exact quantum calculations shown in Figures 2 and 4 provide a stringent test for quasiclassical trajectory simulations of vibrational energy transfer. The comparison of both methods in

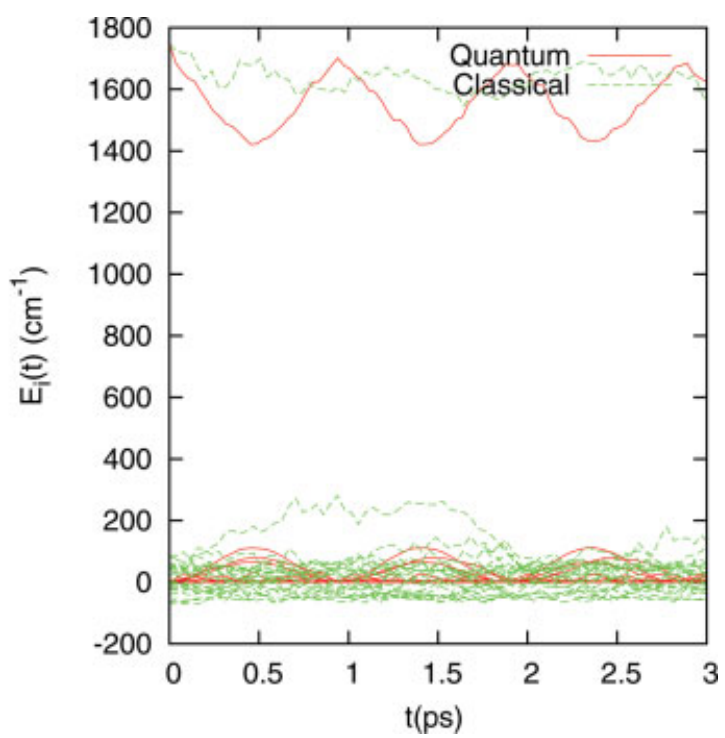

FIGURE 2. Time evolution of the energy content of the initially excited amide I mode and the remaining modes of the NMA. Compared are quantum (red lines) and classical (green lines) calculations, obtained at the DFT/B3LYP (left) and MP2 (right) level of theory. [Color figure can be viewed in the online issue, which is available at www.interscience.wiley.com.] 

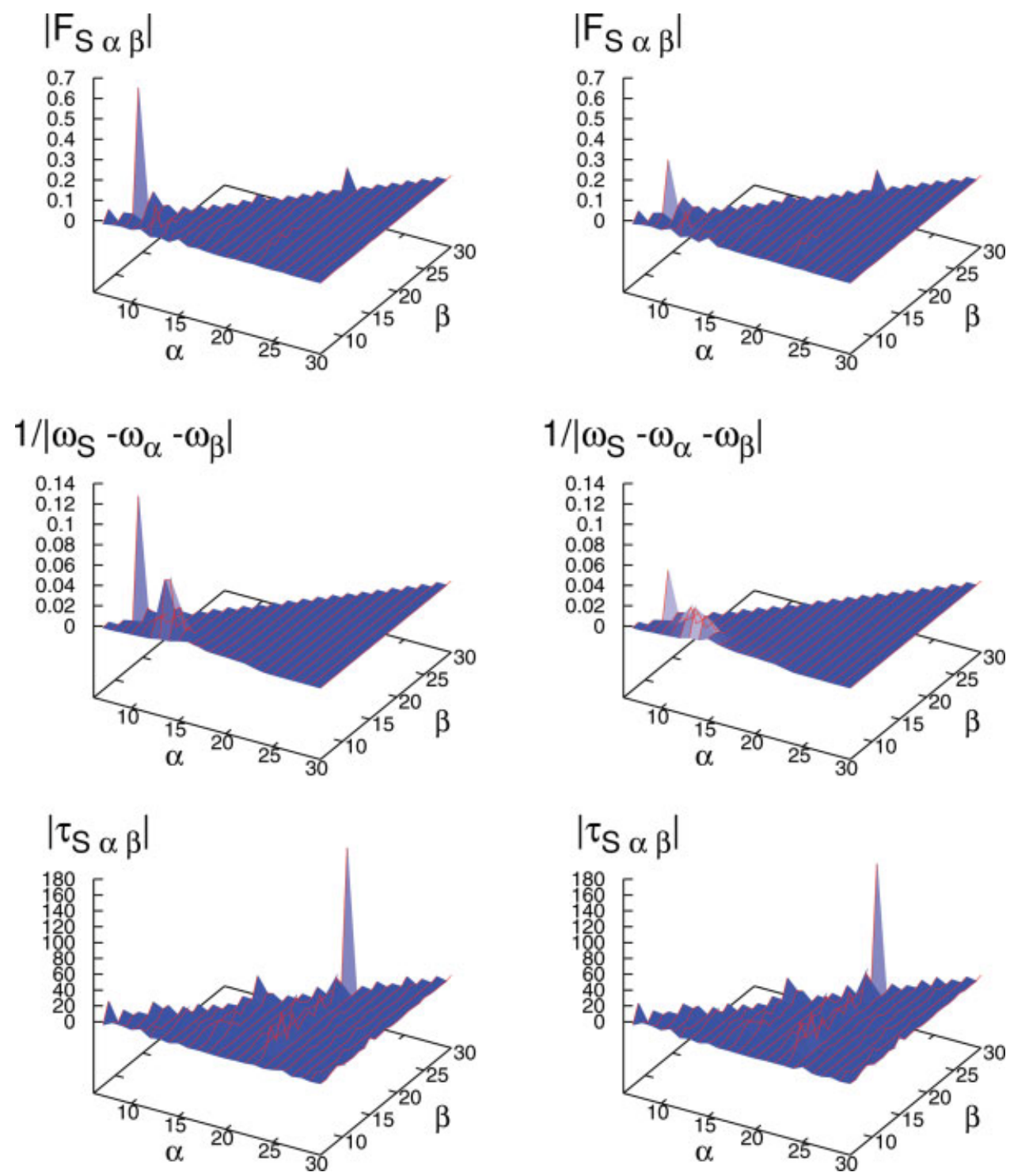

FIGURE 3. Fermi resonance parameter $F_{S_{\alpha \beta}}$ [Eq. (15)] together with its resonance factor $\left|1 /\left(\omega_{S}-\omega_{\alpha}-\omega_{\beta}\right)\right|(\mathrm{cm})$ and the anharmonic coefficient $\left|\tau_{S_{\alpha \beta}}\right|\left(\mathrm{cm}^{-1}\right)$. Compared are quantum calculations, obtained at the DFT/B3LYP (left) and MP2 (right) level of theory. Note that the sharp peaks in $\left|\tau_{S_{\alpha \beta} \beta}\right|$ are due to the self-anharmonicity of the amide I mode. [Color figure can be viewed in the online issue, which is available at www.interscience.wiley.com.]

these figures reveals that for all cases considered the quasiclassical approximation yields at least qualitative agreement with the reference calculation. It is interesting to note, though, that the classical results deviate already at short times. This is a consequence of the fact that we have not employed the full zero point energy in initial conditions (17) and (18). Doing so, we indeed obtain the correct dynamics for the first few hundreds of femtoseconds, but also observe unphysical flow of zero point energy at longer times (data not shown) [19]. By employing full zero point energy in the initially excited amide I mode and thermal energy in all remaining vibrational modes, on the other hand, we obtain a reasonable classical approximation.

Nonetheless, certain care should be taken when a quasiclassical approximation to quantum dynamics is employed. In classical mechanics, energy can flow among the vibrational modes without the restrictions of quantum mechanics. For example, one may observe classical energy transfer, if the initially excited vibrational mode $\omega_{S}$ is about half of the frequency of a high-frequency bath mode $\omega_{\alpha}$ (i.e., $2 \omega_{S} \approx \omega_{\alpha}$ ), even though the initially excited 

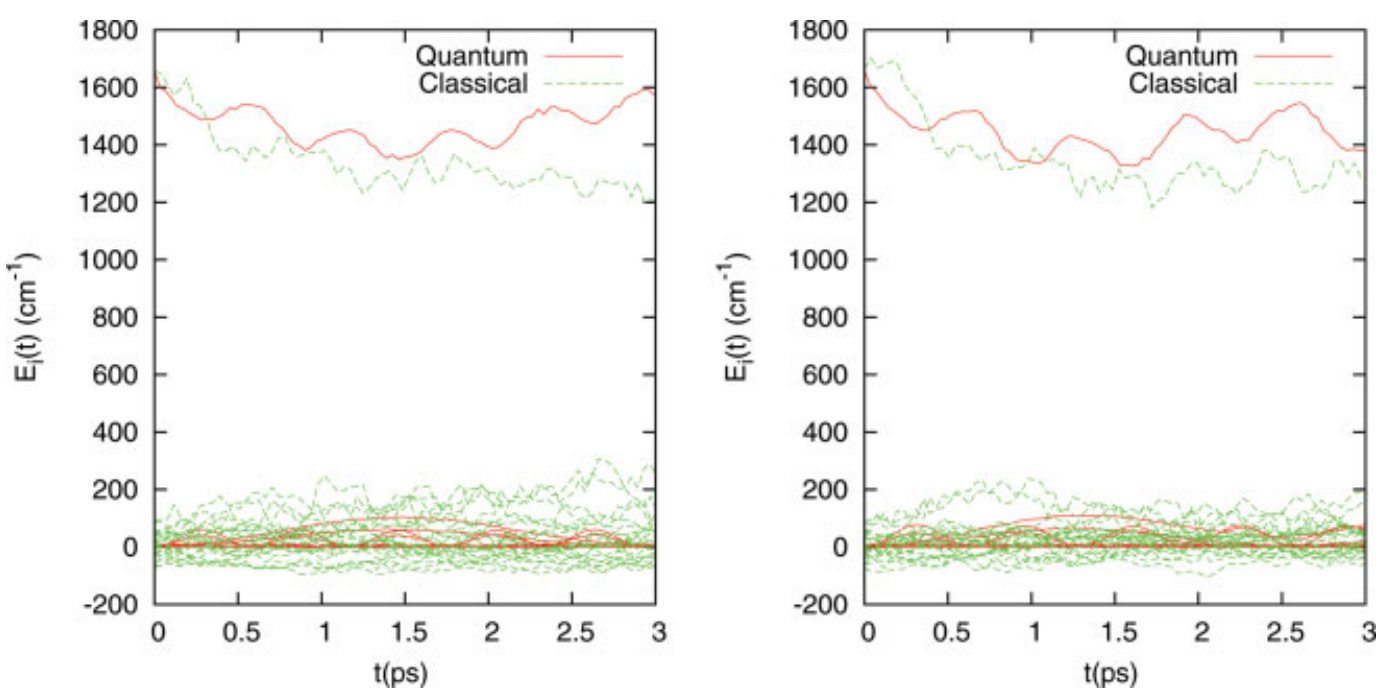

FIGURE 4. Vibrational energy relaxation of NMA, obtained from DFT/B3LYP calculations using an implicit solvent model. Compared are quantum (red lines) and classical (green lines) calculations, obtained for implicit water (left) and implicit methanol (right). [Color figure can be viewed in the online issue, which is available at www.interscience.wiley.com.]

mode contains only the energy of a single quantum. For the present force field, this behavior occurred when the amide II mode was excited and energy transfer to the $\mathrm{C}-\mathrm{H}$ stretch modes was observed (data not shown). As in the case of the zero point energy problem, it is therefore always advisable to check the main energy pathways of classical calculations, in order to make sure that the classical energy flow could also happen in quantum mechanics.

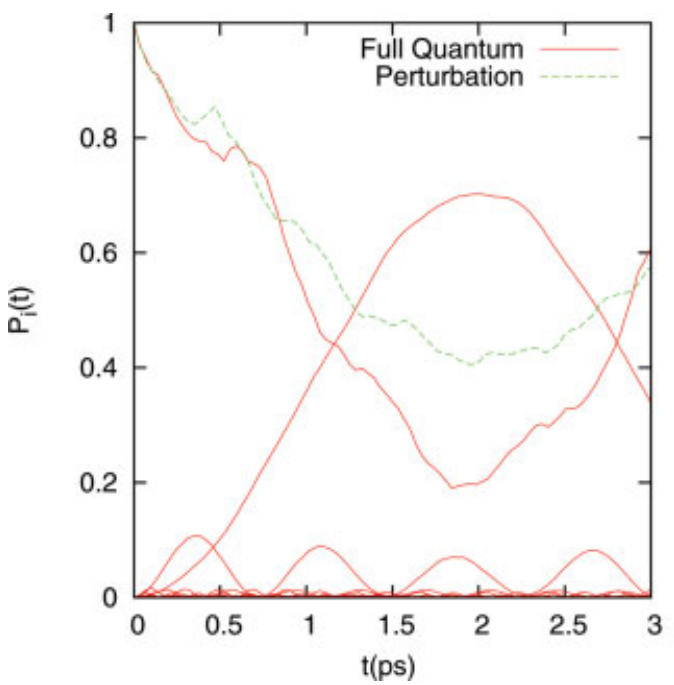

\subsection{PERTURBATIVE DESCRIPTION OF RELAXATION DYNAMICS}

Apart from classical approximations, Golden Rule-type methods as described in Sec. 2.3 have be proven useful to describe vibrational relaxation dynamics [20,42]. The comparison of this method to the reference calculations in Figure 5 reveals that the perturbative expression gives quantitative results for the first few hundreds of femtoseconds. This may be

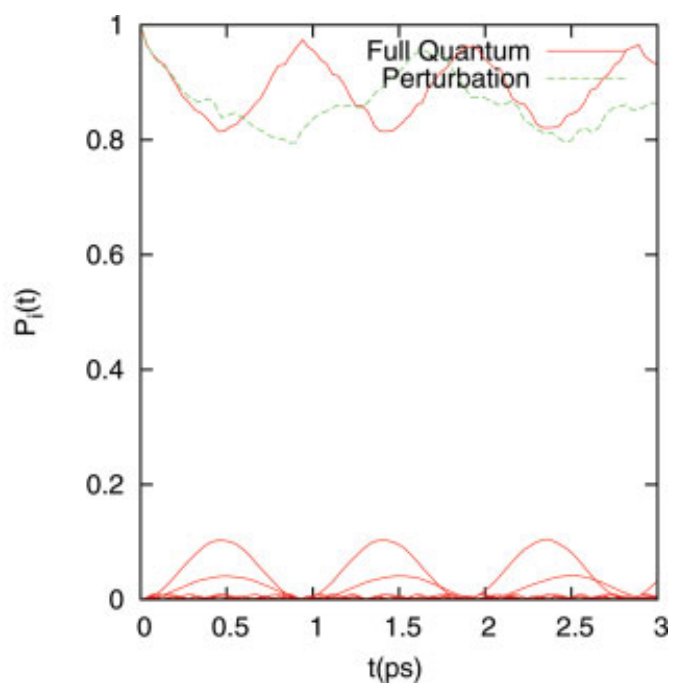

FIGURE 5. Comparison of exact and perturbative calculation of vibrational energy relaxation of the amide I mode of NMA, employing (left) B3LYP and (right) MP2 level of theory. [Color figure can be viewed in the online issue, which is available at www.interscience.wiley.com.] 

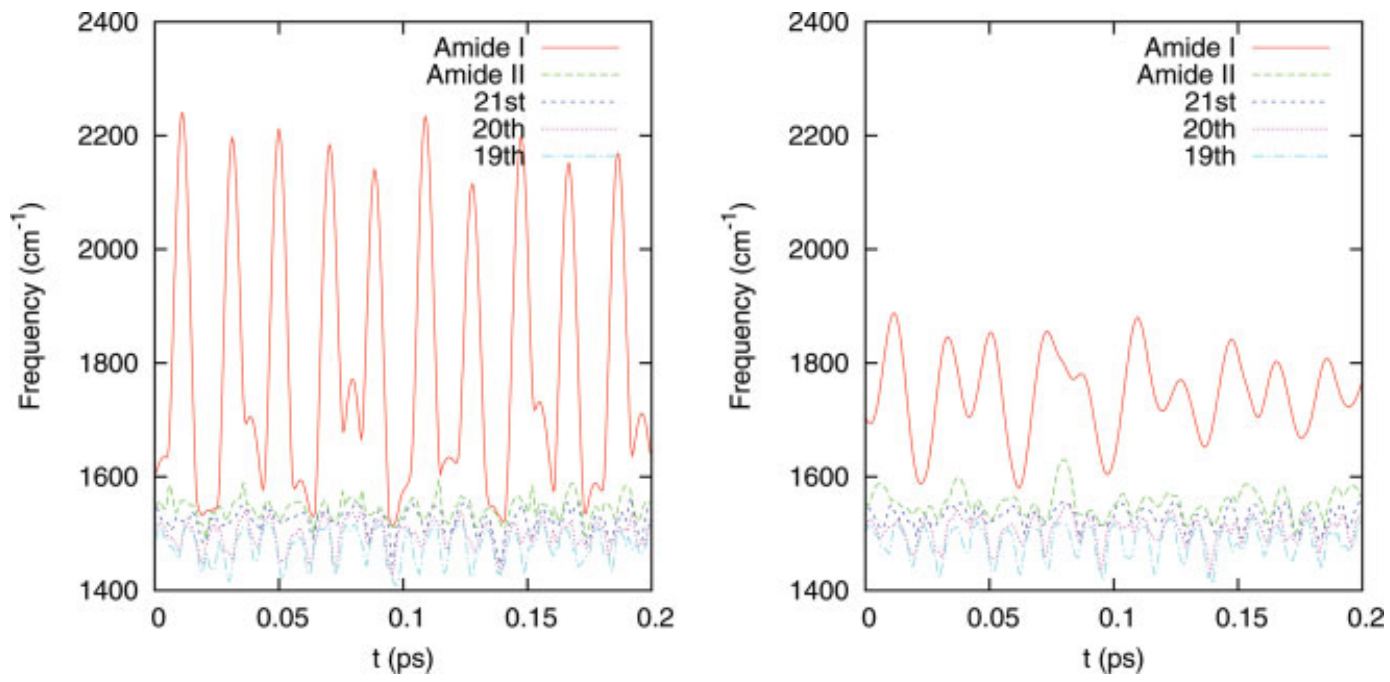

FIGURE 6. Instantaneous normal mode frequencies of selected high-frequency modes of NMA, assuming (left) nonequilibrium and (right) equilibrium initial conditions, respectively. [Color figure can be viewed in the online issue, which is available at www.interscience.wiley.com.]

expected, because the initial process is given by the energy transfer between the amide I mode and the doorway states (or first tiers) [53], which is correctly taken into account by the perturbative formula. At longer times, higher-order interactions and the subsequent relaxation of the doorway states into other degrees of freedom may become important, which is not included in the second-order perturbative description. Nevertheless, in the present case the perturbative calculation yields qualitatively correct results for the first few picoseconds.

A possible approach to extend the application of Golden Rule-type methods to the condensed phase is to take into account the fluctuations of the solvent by calculating the instantaneous normal mode frequencies of the solute [54]. To study the validity of this approach, we have calculated instantaneous normal mode frequencies for the present model, assuming both equilibrium and nonequilibrium initial conditions. As shown in Figure 6, the instantaneous normal mode frequencies of the amide I mode and other high-frequency modes of NMA may undergo fluctuations of several hundred wavenumbers. Even under equilibrium conditions, the amide I mode may vary by more than two hundred wavenumbers. This behavior is quite unrealistic, when we recall that, in experiment, typically fluctuations of tens of wavenumbers are observed [4-6]. We conclude that instantaneous normal mode frequencies cannot be regarded as "true" vibrational frequencies, when strongly anharmonic ab initio PES are employed. A more realistic description of the time-dependent frequencies is obtained, if we perform a geometry optimization of the solute molecule, leading to "quenched normal modes" [44, 54]. Because of the absence of a fluctuating solvent, however, in the present case this procedure only leads to the global minimum of the molecule and therefore to timeindependent (standard) normal mode frequencies.

\section{Conclusions}

Following the previous work [16], we have constructed various quartic force fields of isolated $\mathrm{N}$-methylacetamide (NMA) and performed numerically exact VSCF/VCI calculations of the vibrational energy relaxation of the amide I mode of NMA. All levels of ab initio theory considered (DFT/B3LYP and MP2 with $6-31+G(d)$ or $6-31++G(d, p)$ basis set) reproduced the vibrational frequencies of NMA at least qualitatively. However, the various theoretical levels were found to lead to significant deviations of the vibrational energy relaxation. This behavior could be explained through the Fermi resonance parameter [Eq. (15)], which includes the anharmonic coupling as well as the resonance condition of the energy transfer. The latter was found to predominantly determine the efficiency of the process. It should be noted that this sensitivity to the accurate description of vibrational frequency is particularly high in the case of isolated NMA. Larger peptides and proteins in aqueous solution provide a 
much higher vibrational level density, which facilitates efficient relaxation, since there are always some approximately resonant modes available [42]. Combined with the overall averaging effect of solventinduced frequency fluctuations, this may render a more qualitative quantum-chemical description sufficient.

Taking the VSCF/VCI results as reference calculations, we have studied the validity and accuracy of various approximations usually employed. It was found that second-order perturbation theory provides a faithful description of the first few hundreds of femtoseconds of the relaxation dynamics. This is because the initial energy transfer between the amide I mode and the doorway states is correctly described by the perturbative formula. At longer times, this approximation yields only qualitatively correct results, since higher-order interactions and the subsequent relaxation of the doorway states into other degrees of freedom may become important. To extend this method to the condensed phase, instantaneous normal modes are often invoked in order to account for the solvent-induced frequency fluctuations of the system. In the present case of strongly anharmonic ab initio PES, however, the resulting instantaneous normal mode frequencies were found to undergo unrealistically high fluctuations of several hundred wavenumbers. A more realistic description of the time-dependent frequencies may be obtained, if we perform a geometry optimization of the solute molecule, leading to "quenched normal modes" [44, 54].

We have also performed quasiclassical trajectory simulations of vibrational energy transfer, which were found to be in at least qualitative agreement with the reference calculation. However, in classical mechanics, energy can flow among the vibrational modes without the restrictions of quantum mechanics. A well-known example is the zero-point energy problem, which in the present case was circumvented by employing full zero point energy only in the initially excited amide I mode and thermal energy in all remaining vibrational modes. Moreover, in classical mechanics energy transfer may also occur to high-frequency bath modes. To assure that the classical energy flow also would happen in quantum mechanics, it is therefore always advisable to check the main energy pathways of a classical calculation.

\section{ACKNOWLEDGMENTS}

The authors thank P.H. Nguyen, S.M. Park, and A. Dreuw for discussions and helpful comments. H.F. deeply appreciates Prof. Kimihiko Hirao's hospitality and generosity during H.F.'s visit to Prof. Hirao's laboratory.

\section{References}

1. Krimm, S.; Bandekar, J. Adv Prot Chem 1986, 38, 181.

2. Barth, A.; Zscherp, C. Q. Rev Biophys 2002, 35, 369.

3. Torii, H.; Tasumi, M. J Chem Phys 1992, 96, 3379.

4. Hamm, P.; Lim, M. H.; Hochstrasser, R. M. J Phys Chem B 1998, 102, 6123.

5. Zanni, M. T.; Asplund, M. C.; Hochstrasser, R. M. J Chem Phys 2001, 114, 4579.

6. DeFlores, L. P.; Ganim, Z.; Ackley, S. F.; Chung, H. S.; Tokmakoff, A. J Phys Chem B 2006, 110, 18973.

7. Torii, H.; Tasumi, M. J Raman Spec 1998, 29, 81.

8. Gregurick, S. K.; Chaban, G. M.; Gerber, R. B. J Phys Chem A 2002, 106, 8696 .

9. Schmidt, J. R.; Corcelli, S. A.; Skinner, J. L. J Chem Phys 2004, $121,8887$.

10. Ham, S.; Hahn, S.; Lee, C.; Cho, M. J Phys Chem B 2005, 109, 11789.

11. Hayashi, T.; Jansen, T. 1. C.; Zhuang, W.; Mukamel, S. J Phys Chem A 2005, 109, 64.

12. Bounouar, M.; Scheurer, Ch. Chem Phys 2006, 323, 87.

13. (a) Gorbunov, R. D.; Nguyen, P. H.; Kobus, M.; Stock, G. J Chem Phys 2007, 126, 054509; (b) Kobus, M.; Gorbunov, R. D.; Nguyen, P. H.; Stock, G. Chem Phys 2008, 347, 208.

14. Kaledin, A. L.; Bowman, J. M. J Phys Chem A 2007, 111, 5593.

15. (a) Leitner, D. M. Adv Chem Phys 2005, 130B, 205; (b) Leitner, D. M. Annu Rev Phys Chem 2008, 59, 233.

16. Fujisaki, H.; Yagi, K.; Hirao, K.; Straub, J. E. Chem Phys Lett 2007, 443, 6 .

17. Dijkstra, A. G.; Jansen, T. 1. C.; Bloem, R.; Knoester, J. J Chem Phys 2007, 127, 194505.

18. Pouthier, V. J Chem Phys 2008, 128, 065101.

19. Nguyen, P. H.; Stock, G. J Chem Phys 2003, 119, 11350.

20. Fujisaki, H.; Zhang, Y.; Straub, J. E. J Chem Phys 2006, 124, 144910.

21. (a) Brooks, B. R.; Bruccoleri, R. E.; Olafson, B. D.; States, D. J.; Swaminathan, S.; Karplus, M. J Comp Chem 1983, 4, 187; (b) MacKerell, A.D. Jr.; Brooks, B.; Brooks, C. L., III; Nilsson, L.; Roux, B.; Won, Y.; Karplus, M. The Encyclopedia of Computational Chemistry 1: Schleyer, P. v. R., et al., Eds.; Chichester: Wiley, 1998; pp. 271-277.

22. van der Spoel, D.; Lindahl, E.; Hess, B.; Groenhof, G.; Mark, A. E.; Berendsen, H. J. C. J Comp Chem 2005, 26, 1701.

23. Bowman, J. M. J Chem Phys 1978, 68, 608.

24. Carter, S.; Bowman, J. M.; Handy, N. C. Theor Chem Acc 1998, 100, 191.

25. Bowman, J. M.; Carter, S.; Huang, X. Int Rev Phys Chem 2003, 22,533 .

26. (a) Gatti, F.; Meyer, H.-D. Chem Phys 2004, 304, 3; (b) Kühn, O. Chem Phys Lett 2005, 402, 48. 
27. (a) Egorov, S. A.; Skinner, J. L. J Chem Phys 1996, 105, 8973; (b) Egorov, S. A.; Everitt, K. F.; Skinner, J. L. J Phys Chem A 1999, 103, 9494; (c) Lawrence, C. P.; Skinner, J. L. J Chem Phys 2002, $117,5827$.

28. (a) Stratt, R. M. Acc Chem Res 1995, 28, 201; (b) Keyes, T. J Phys Chem A 1997, 101, 2921.

29. Giese, K.; Petkovic, M.; Naundorf, H.; Kühn, O. Phys Rep 2006, 430, 211.

30. Yagi, K.; Hirata, S.; Hirao, K. Theo Chem Acc 2007, 118, 681.

31. Rauhut, G. J Chem Phys 2004, 121, 9313.

32. (a) Kongsted, J.; Christiansen, O. J Chem Phys 2006, 125, 124108; (b) Toffoli, D.; Kongsted, J.; Christiansen, O. J Chem Phys 2007, 127, 204106.

33. (a) Yagi, K.; Hirata, S.; Hirao, K. J Chem Phys 2007, 127, 034111; (b) Yagi, K.; Taketsugu, T.; Hirao, K.; Gordon, M. S. J Chem Phys 2000, 113, 1005.

34. Carter, S.; Culik, S. J.; Bowman, J. M. J Chem Phys 1997, 107, 10458.

35. Yagi, K.; Hirao, K.; Taketsugu, T.; Schmidt, M. W.; Gordon, M. S. J Chem Phys 2004, 121, 1383.

36. Barone, V. J Chem Phys 2005, 122, 014108.

37. Taketsugu, T.; Yagi, K.; Gordon, M. S. Int J Quantum Chem $2005,104,758$.

38. Krishnan, G. M.; Kühn, O. Chem Phys Lett 2007, 435, 132.

39. Frisch, M. J.; Trucks, G. W.; Schlegel, H. B.; Scuseria, G. E.; Robb, M. A.; Cheeseman, J. R.; Montgomery, Jr., J. A.; Vreven, T.; Kudin, K. N.; Burant, J. C.; Millam, J. M.; Iyengar, S. S.; Tomasi, J.; Barone, V.; Mennucci, B.; Cossi, M.; Scalmani, G.; Rega, N.; Petersson, G. A.; Nakatsuji, H.; Hada, M.; Ehara, M.; Toyota, K.; Fukuda, R.; Hasegawa, J.; Ishida, M.; Nakajima, T.; Honda, Y.; Kitao, O.; Nakai, H.; Klene, M.; Li, X.; Knox, J. E.; Hratchian, H. P.; Cross, J. B.; Bakken, V.; Adamo, C.; Jaramillo, J.; Gomperts, R.; Stratmann, R. E.; Yazyev, O.; Austin, A. J.; Cammi, R.; Pomelli, C.; Ochterski, J. W.; Ayala, P. Y.; Morokuma, K.; Voth, G. A.; Salvador, P.; Dannenberg, J. J.;
Zakrzewski, V. G.; Dapprich, S.; Daniels, A. D.; Strain, M. C.; Farkas, O.; Maiick, D. K.; Rabuck, A. D.; Raghavachari, K.; Foresman, J. B.; Ortiz, J. V.; Cui, Q.; Baboul, A. G.; Clifford, S.; Cioslowski, J.; Stefanov, B. B.; Liu, G.; Liashenko, A.; Piskorz, P.; Komaromi, I.; Martin, R. L.; Fox, D. J.; Keith, T.; Al-Laham, M. A.; Peng, C. Y.; Nanayakkara, A.; Challacombe, M.; Gill, P. M. W.; Johnson, B.; Chen, W.; Wong, M. W.; Gonzalez, C.; Pople, J. A. Gaussian 03, Revision C.02, Gaussian, Inc., Wallingford CT, 2004.

40. Yagi, K. SINDO Version 1.3, 2006.

41. Bowman, J. M. Acc Chem Res 1986, 19, 202.

42. Fujisaki, H.; Straub, J. E. J Phys Chem B 2007, 111, 12017.

43. Cremeens, M.; Fujisaki, H.; Zhang, Y.; Zimmermann, J.; Sagle, L. B.; Matsuda, S.; Dawson, P. E.; Straub, J. E.; Romesberg, F. E. J Am Chem Soc 2006, 128, 6028.

44. (a) Ohmine, I.; Tanaka, H. J Chem Phys 1990, 93, 8138; (b) Ohmine, I.; Tanaka, H. Chem Rev 1993, 93, 2545.

45. (a) Moritsugu, K.; Miyashita, O.; Kidera, A. Phys Rev Lett 2000, 85, 3970; (b) Moritsugu, K.; Miyashita, O.; Kidera, A. J Phys Chem B 2003, 107, 3309.

46. (a) Voth, G. A.; Marcus, R. A. J Chem Phys 1985, 82, 4064; (b) Ka, B. J.; Geva, E. J Phys Chem A 2006, 110, 13131.

47. Schinke, R. Photodissociation Dynamics; Cambridge University Press: Cambridge, 1993.

48. Ankerhold, J.; Saltzer, M.; Pollak, E. J Chem Phys 2002, 116, 5925.

49. Yoshida, H. Phys Lett A 1990, 150, 262.

50. Guo, Y.; Thompson, D. L.; Sewell, T. D. J Chem Phys 1996, 104, 576 .

51. (a) Stock, G.; Müller, U. J Chem Phys 1999, 111, 65; (b) Müller, U.; Stock, G. J Chem Phys 1999, 111, 77.

52. Ataka, S.; Takeuchi, H.; Tasumi, M. J Mol Struct 1984, 113, 147.

53. (a) Gruebele, M. J Phys: Condens Matter 2004, 16, R1057; (b) Gruebele, M.; Wolynes, P. G. Acc Chem Res 2004, 37, 261.

54. Fujisaki, H.; Stock, G. J Chem Phys 2008, 129, 134110. 